\title{
Editorial
}

\section{Article Keyword Selection}

Keyword selection is the second Sudan Journal of Medical Sciences (SJMS) short guidance piece on the international academic standards. As a Section Editor at SJMS, I am honored to be invited to write in this series. In future, I can see SJMS leading the way to improving the publishing standards in Sudan's medical field. And with the current practice and unique services that SJMS provides for their readers and authors, it marks its step to be one of the best regional journals in the discipline.

\section{The Importance of Keywords}

As authors, we aspire to share our work among the readers in our field and get appropriately cited. Here comes the volume for the authors' keywords. The first journey for each manuscript begins with a literature review in which the indexing databases such as Scopus and PubMed are searched for any present work related to our ideas. This ends by the citation of most of those works in our reference list. It is curial to select

Received 15 September 2020 Accepted 25 September 2020

Published

30 September 2020

Production and Hosting by

Knowledge E

Editor-in-Chief:

Prof. Mohammad A. M. Ibnouf your keywords so that it acts as a promoter for your work among your colleagues. Apart from being used by the indexing service to classify your work, keywords for the publisher and the editorial board serve as a guidance for choosing the referee for your article and your paper's subject.

\section{How to choose your keyword?}

1. First, make a list of words from the manuscript, which you believe represents the work's essential idea. They could also be a phrase (e.g., colon cancer). Most researchers use sentences or phrases in search engines. Do not use words from your title, as this will decrease the chances of the article appearing during the logarithmic search. There is also a word generator engine like MeSH on Demand, a google keyword planner. Each journal has its own maximum number of keywords; for SJMS, the number of keywords are specified according to the article types.

2. Next, have a look at the standard term used for indexing in the medical field (MeSH, Psyclnfo, and others) and ensure that the keyword of your choice is listed there. 
3. Lastly, go through the standard search engine used commonly by professionals in your field and type it to see if the same titles as yours come up.

To publish means to be known. I believe each article's minor part deserves the same input as a significant section.

Dr. Abduelbagi D.A Altayb, MRCP.

Section Editor, SJMS

aaaldesogi@icloud.com 